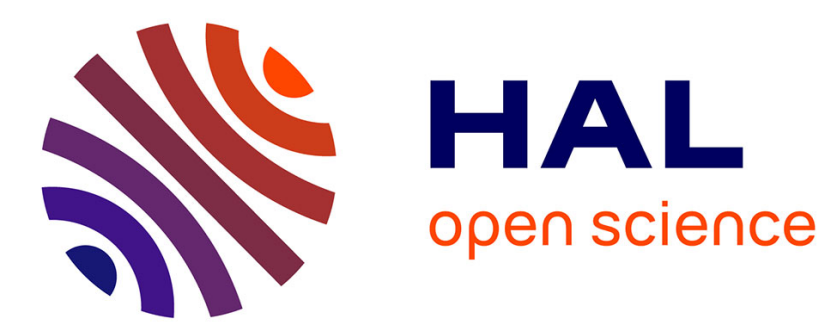

\title{
Adaptive-Segmentation and Flexible-Delay Based Broadcasting Protocol for VANETs
}

Houda Hafi, Wahabou Abdou, Salah Merniz

\section{To cite this version:}

Houda Hafi, Wahabou Abdou, Salah Merniz. Adaptive-Segmentation and Flexible-Delay Based Broadcasting Protocol for VANETs. 15th International Conference on Wired/Wireless Internet Communication (WWIC), Jun 2017, St. Petersburg, Russia. pp.207-218, 10.1007/978-3-319-61382-6_17 . hal-01675423

\section{HAL Id: hal-01675423 \\ https://inria.hal.science/hal-01675423}

Submitted on 4 Jan 2018

HAL is a multi-disciplinary open access archive for the deposit and dissemination of scientific research documents, whether they are published or not. The documents may come from teaching and research institutions in France or abroad, or from public or private research centers.
L'archive ouverte pluridisciplinaire HAL, est destinée au dépôt et à la diffusion de documents scientifiques de niveau recherche, publiés ou non, émanant des établissements d'enseignement et de recherche français ou étrangers, des laboratoires publics ou privés. 


\title{
Adaptive-Segmentation and Flexible-Delay Based Broadcasting Protocol for VANETs
}

\author{
Houda Hafi ${ }^{1}$, Wahabou Abdou ${ }^{2}$, and Salah Merniz ${ }^{1}$ \\ 1 MISC Laboratory, Abdelhamid Mehri University, Constantine, Algeria \\ \{houda.hafi, salah.merniz\}@univ-constantine2.dz \\ 2 LE2I UMR6306, CNRS, Arts et Métiers, Univ. Bourgogne Franche-Comté, F-21000 \\ Dijon, France \\ wahabou . abdou@u-bourgogne.fr
}

\begin{abstract}
A Vehicular Ad hoc Network (VANET) is an interconnection of vehicles that communicate through wireless technologies. It offers to road users a wide variety of applications which can be classified into four main categories: safety, road traffic, comfort and infotainment. This paper deals with safety applications. Their main goal is to detect critical road conditions (e.g. accidents, black ice, etc.) and/or send notifications to other vehicles in the network. An effective dissemination of such a message relies on multi-hop retransmissions. Thus an explicit or implicit cooperation between vehicles is needed in order to relay the message over a wide area. The main challenge is to avoid the broadcast storm problem. This paper proposes an efficient segment-delay based method that divides the road into several segments depending on the network density and utilises a waiting time update technique to expedite the dissemination process with respect to network performance.
\end{abstract}

Keywords: VANETs, Safety applications, Broadcasting protocol, Road segmentation, Delay adjustment.

\section{Introduction}

Reducing the risk of road accidents is a major challenge. Throughout the world, several solutions are proposed to achieve this goal. Some of them take advantage of technological advances, particularly in the field of intelligent transportation systems (ITS) since some ITS's applications rely on interactions between smart vehicles and their environment (another vehicle, a road side unit or any other intelligent node). Compared to a simple car, a smart vehicle is equipped with a set of wireless communication devices such as sensors, radars, global navigation satellite systems (GNSS) like the Global Positioning System (GPS), human machine interfaces, etc. A collaborative multi-hop wireless communication between smart vehicles leads to a Vehicular Ad hoc Network (VANET). Two main communication modes are possible. The first one is an ad hoc mode called vehicle to vehicle $(\mathrm{V} 2 \mathrm{~V})$ communication, where vehicles do not use any pre-existing network infrastructure to communicate. In the second mode, vehicles communicate 
with a fixed devices installed along the road, forming a vehicle to infrastructure (V2I) or infrastructure to vehicle (I2V) communications.

VANETs differ markedly from other types of networks (mobile ad hoc networks - MANETs, sensor networks, mesh networks, etc.) on several points. The first difference rises on mobility features. In MANETs, the nodes speed is that the users walking, whereas, in VANETs the speed is that of the vehicle that can exceed one hundred kilometres per hour. High mobility makes a rapidly changing topology and frequent disconnections between nodes in the network. Moreover, the number of nodes in VANETs is very large compared to MANETs. Furthermore, displacement environment is quite different from that of MANETs [1].

Vehicular networks provide safety applications which aim to protect drivers from any road hazard. These applications need a very short end to end delay, because of the imminence of the danger. Moreover, after a while, the information will become obsolete [2]. To meet this requirement, inter-vehicle communication protocols must take into consideration the network topology and select appropriate relay nodes in order to avoid the broadcast storm problem which leads to an increase in the number of redundant messages, the latency and the radio interferences [3].

This paper proposes a novel broadcasting method which is based on road segmentation. The idea is to privilege the nodes that are farthest from the source without penalising those which are not far away from it if the former have not received the packet due to a collision or fading channel problem.

The remaining of this paper is organised as follows. Section II presents some related works. Section III introduces our broadcasting technique. Simulation results are presented in Section IV. And Section V concludes the paper and point out some future work.

\section{Related Works}

In literature, many solutions have been proposed to tackle the broadcast problems in VANETs. They can be classified into two categories: sender-oriented and receiver-oriented protocols. In the first class, the relay nodes are selected by the sender. In the second one, when a node receives a packet, it decides autonomously if it broadcasts the packet or no.

Several techniques are used to forward emergency packets: knowledge-based, counter-based, probabilistic based, segment-based, delay-based and distancebased. The simplest broadcasting method is the Simple flooding. Every packet is relayed exactly once by each node. An inconvenience of this method is that it may lead to many useless redundant packets since it does not take into account the network topology and density. To overcome this issue, some neighbor knowledge-based protocols have been proposed. These methods are based on a comparison of lists of neighbors: 1-hop neighbor list for Distributed Vehicular Broadcast (DV-CAST) [4] or 2-hop neighbor list for Scalable Broadcast Algorithm (SBA) [5]. These lists are included in the broadcast packets and help receivers to check if it is useful to retransmit the packets (if there exist addi- 
tional nodes that may receive the packet). However, this makes the data packet size very large especially in a dense network.

Another category of broadcasting protocols is the counter-based methods. They rely on the idea that the more a node receives copies of the packet, the less likely it is useful to relay this packet. Upon reception of the first copy, the node initialises a counter and sets a timeout. During the waiting time, the counter is incremented upon reception of a copy of the packet. At the expiration of the waiting period, the packet is retransmitted if the counter of redundant copies is less than a threshold value. AckPBSM [6] and POCA [7] use this approach and set lower timeout to the farthest nodes from the source (or last-hop relay).

Probabilistic-based methods represent a further receiver-oriented dissemination technique to broadcast an emergency message in VANETs. In these schemes, the receiver calculates a dissemination probability based on a defined parameter. For instance, authors in [8] associate the forwarding probability of a node to its distance from the source so that the farthest node will have a high chance to rebroadcast the packet. In [9-11] the rebroadcasting probability depends on the node's local density, node's speed and redundancy ratio respectively. In [12] authors propose a protocol called E-ProbT that combines the number of common neighbors and the distance between the transmitter and the receiver to compute the probability of forwarding.

To resolve the broadcast storm problem and have a high reliability, authors in [13-15] use the segment-based technique. They divide the road into multiple segments and assign the responsibility of dissemination and acknowledgement of the broadcast message to the farthest vehicle in the last non-empty segment.

To deal with rebroadcast redundancy and provide an appropriate end to end delay which is a critical quality of service requirement in safety applications, several protocols that are based on delay-based schemes have been proposed. In this strategy, distant nodes from the source have the lowest waiting time. Therefore, they have the highest priority to rebroadcast the packet. Slotted 1persistence [8] is a delay-based protocol where all nodes located in the same segment will have an identical waiting time. Although, it privileges the farthest nodes by giving them the shortest slot time, the collision problem can not be circumvented, on account of the simultaneous access to the radio channel by all the nodes that belong to the same segment.

Distance-based schemes represent the most used by all aforementioned techniques since all protocols have the same end, viz. reliable and fast data packet delivery with a few hop counts. The main idea behind this technique is that the source uses the information gained from periodic hello messages and choose the farthest node as a forwarder [16,17]. In [18] authors exhibit an amendment sender-oriented distance-based protocol, where the source pick the effective forwarders and incorporate them in the data packet header. Receiving the packet, the node checks whether it has been chosen as relay node. In the positive case, it calculates its relay waiting time which is defined as $\alpha \times i$, where $i$ is the node's index in the list of forwarder candidates and $\alpha$ is a pre-defined slot time. In their work, they assume that $\alpha$ is $10 \mathrm{~ms}$ and the number of relay nodes is 
12 (from 0 to 11). If the receiver is not part of the forwarder candidates, the packet is discarded.

\section{Dynamic Segment-Delay Broadcasting Protocol}

There are two approaches to broadcast a safety message in VANETs: senderoriented and receiver-oriented methods. The reliability of each technique depends on the transmission conditions. In the case of obstacle-free environment that allows a direct vision (Line Of Sight) between the transmitter and the receiver, the sender-oriented method may be preferred. However, its performances degrade quickly in a complex environment where there are many obstacles that hinder the errorless reception of the message by the relay nodes falling further in distance from the source. In this case, it's better that each node decides in an autonomous way. Nevertheless, if all receivers decide to rebroadcast the packet at the same time, this involves many redundant packets which will saturate the bandwidth and consequently increase the collisions and the latency. Therefore, a hybrid technique is advisable to avail of the advantages of each one.

This paper introduces a new distributed broadcast technique called SegmentDelay Based Broadcast Protocol (SDBP) for vehicular ad hoc networks. Our proposed combines the sender-oriented and receiver-oriented schemes. SDBP is sender-oriented in the sense that the source helps the nodes with some information that incorporates in the data packet header, and receiver-oriented where each node acts autonomously after receiving the data packet.

\subsection{An overview of the proposed method}

The aim of SDBP is to guarantee a high and fast dissemination of a safety message in a sparse and high-density network. It is based on segment and delay dissemination techniques. It gives the high priority of forwarding to the nodes located in the farthest segment. The novelty of our work relies on the dynamic adaptation of the waiting time of each node after the ending of the fixed period referred to "SlotTime" for which a packet will be received by the other nodes. We devote following sections below to delineate the different steps of SDBP.

\subsection{Sending Phase}

The sending process may be triggered by a vehicle that detects an unexpected road hazard. In such case, it should inform all its neighbors by broadcasting an emergency message. To ensure the reliability of message dissemination in a sparse and dense network, SDBP proposes a dynamic density-based segmentation technique. Most of the segmentation techniques proposed in literature produce the empty segment problem $[8,13,15]$. In general, the length of each segment is obtained by dividing the communication range of the source node by the desired segment number. In such case, if the nodes are not uniformly distributed, the farthest segments may be empty. Some techniques are proposed to 
remedy this problem. SDBP introduces a new approach that completely avoids the appearance of empty segments. In SDBP, the number of segment relies on the local density of a node (number of neighbors) and it does not depend on node's transmission range which is unknown by the sender in the real world since it is inconstant and varies swiftly depending on the signal propagation environment.

To better manage the groups of vehicles and facilitate the task of relay nodes selection, the source divides its transmission range into $K$ segments depending on its number of neighbors $(N)$.

$$
K=\lfloor\sqrt{N}\rceil
$$

All segments are uniform in terms of number of nodes $M_{i}, i \in\{1,2, . . k\}$, excluding the farthest segment. Indeed, when $N$ is not a multiple of $K$, the farthest segment will have a high number of nodes compared to the other segments. this will increase the number of nodes with a high priority in broadcasting. Equation 2 is used to determine the number of nodes in each segment.

$$
M_{i}= \begin{cases}\frac{N}{K}+(N \bmod K) & \text { if } \mathrm{i}=1 \text { for the farthest segment } \\ \frac{N}{K} & \text { for } \mathrm{i} \in\{2,3, . . k\}\end{cases}
$$

In order to reduce the dissemination time of each message, priority of relaying messages should be given to nodes that are in the farthest segment. Therefore, each node must be aware of the segment it belongs to. The maximum and minimum boundaries of each segment are included in the data packet header (see figure 1). Equation 3, explains how to reckon the boundaries of each segment.

\begin{tabular}{|c|c|c|c|c|c|c|c|}
\hline Broadcaster ID & $\begin{array}{c}\text { The number of } \\
\text { segments }\end{array}$ & $\begin{array}{c}\text { The farthest } \\
\text { node's position }\end{array}$ & Dmin $_{1}$ & Dmin $_{2}$ & $\ldots \ldots \ldots . .$. & Dmin $_{\mathrm{k}}$ & DATA \\
\hline
\end{tabular}

Fig. 1. SDBP Packet Header

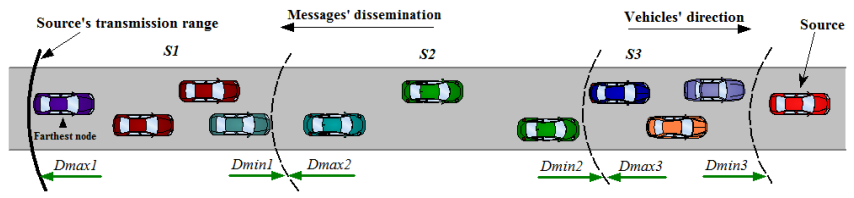

Fig. 2. Example of segmentation process 


$$
\left\{\begin{aligned}
n_{i} & =(N+1)-\sum_{j=1}^{i} M_{j} \\
\operatorname{Dmin}_{i} & =n_{i}^{\text {th }} \text { neighbor's position } \\
\text { Dmax }_{i} & = \begin{cases}\text { Farthest neighbor's position } & \text { if } \mathrm{i}=1 \\
\text { Dmin }_{i-1} & \text { if } \mathrm{i} \in\{2,3, . . k\}\end{cases}
\end{aligned}\right.
$$

\subsection{Forwarding Phase}

Figure 3 summarises the main idea of SDBP that can be explained as follows: When a node receives a data packet for the first time, it initialises a counter to compute the number of redundant copies. Then, it determines to which segment it belongs to and calculates its waiting time $W T$ according to Equation 4.

$$
W T_{j}=\alpha \times \text { SlotTime } \times \frac{d(\text { Source }, \text { Farthest Node })}{d(j, \text { Source })}
$$

where $\alpha \in\{1,2, \ldots, K\}$ is the segment's number, $d$ represents the distance between two nodes and SlotTime is an approximate time for a packet to be thoroughly received by the other neighbors. In our work, we have taken the SlotTime value proposed in [11].

Afterwards, a timer is triggered which is the minimum between waiting time and SlotTime. At the timer's expiration, three cases may be distinguished:

- If the node has already received another copy of the same packet, this latter will be discarded.

- If no copy has been received by the node and its waiting time is expired $(W T=0)$, the node forwards the packet.

- If the waiting time of the node has not expired yet and it is always greater than SlotTime, the node updates its waiting time through the Equation 5. Else, the minimum value between $W T$ and SlotTime is selected again and the process restarts.

$$
W T_{j}^{\prime}=\frac{W T_{j}}{2}
$$

The reasoning behind the waiting time adjustment is to hasten the dissemination process mostly in a network where there are many obstructions that prevent the good reception of the message. In actuality, when a node broadcasts a safety message, it does not know which nodes will receive the packet and which ones will lose it. For instance, in a network where the message is lost by all nodes falling farther from the source, the nearest neighbors to the source must wait a lengthy time before broadcasting the message. This approach enormously increases the end to end delay. 


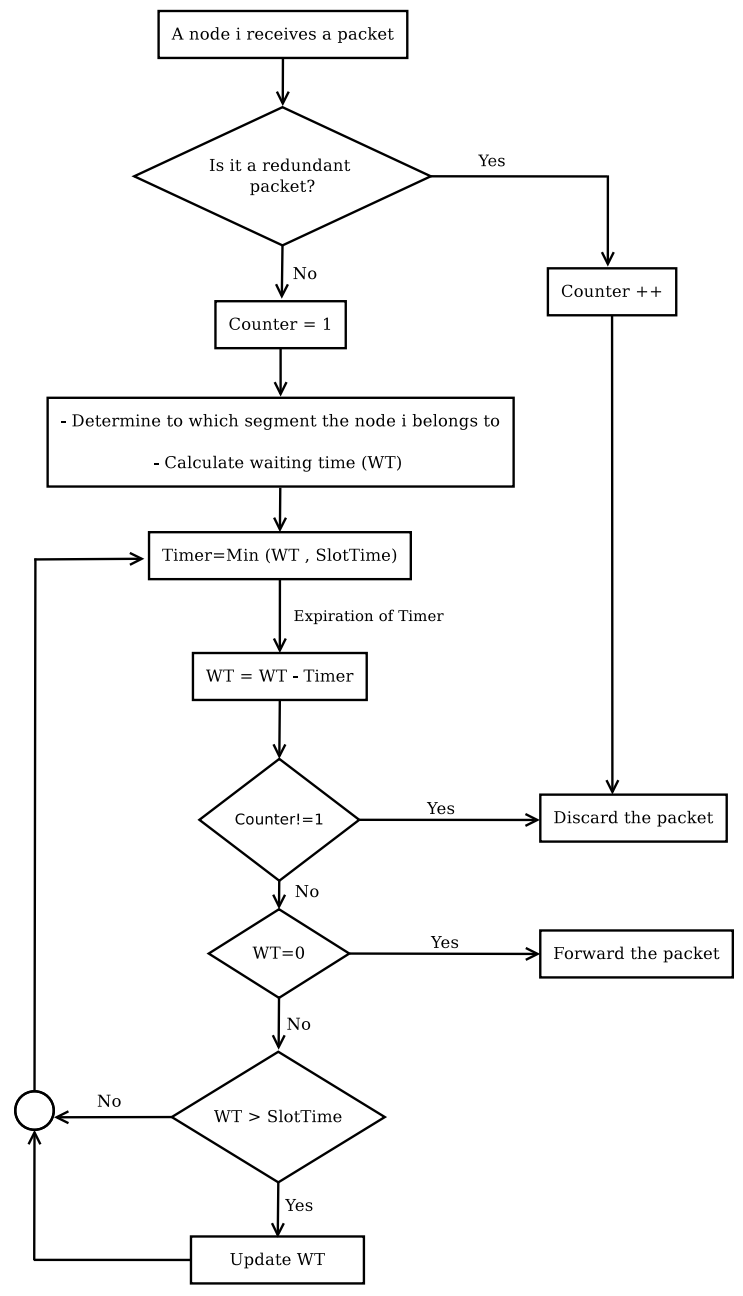

Fig. 3. Forwarding process of SDBP

\section{Experimentation and Results}

To assess the performance of SDBP, we carried out our simulations thanks to Network Simulator 2 (NS2, version 2.35) [19]. We have used the m-Nakagami propagation model and IEEE $802.11 \mathrm{p}$ as wireless communication standard. To generate mobility traces, we have used a microscopic traffic simulator called "SUMO" [20]. The simulation starts by broadcasting a safety message from the vehicle located at the extremity of the road. In order to evaluate SDBP under different density environments (sparse, moderate and dense), we vary the number and the speed of vehicles in the network according to Table 2 so that the denser the network the smaller the speed of vehicles. The maximum speed in each 
scenario is $110 \%$ of the minimum speed provided in Table 2. Each simulation scenario is run 10 times and the results presented in this section are the average values of the performance metrics detailed in section 4.1 . The detailed simulation settings are given in Table 1.

Table 1. simulation scenarios parameters

\begin{tabular}{ll}
\hline Network Simulator & NS-2.35 \\
Traffic and Mobility Generator & SUMO 0.28.0 \\
Highway Lenght & $8 \mathrm{Km}$ \\
MAC protocol & IEEE $802.11 \mathrm{p}$ \\
Number of data packet sources & 1 \\
SlotTime & $4 \mathrm{~ms}$ \\
Number of data packets & 30 \\
Propagation model & m-Nakagami \\
\hline
\end{tabular}

Table 2. speed values

\begin{tabular}{|c|c|c|c|c|c|c|c|}
\hline Vehicles density(vehicles/km) & 10 & 20 & 30 & 50 & 60 & 90 & 120 \\
\hline Minimum speed of vehicles $(\mathrm{m} / \mathrm{s})$ & 20 & 10 & 7 & 4 & 3 & 2 & 1.5 \\
\hline
\end{tabular}

To validate the performance of SDBP, two protocols are chosen namely: the Simple Flooding which is a reference protocol and the Furthest Distance to show the behavior of a protocol based solely on the sender-oriented technique in a non-deterministic environment.

- Simple Flooding Protocol (SFP): the well-known technique where nodes broadcast each received packet only once (the first time) without any waiting time.

- Furthest Distance Protocol (FDP): a sender-oriented distance-based dissemination technique where the duty of forwarding is ascribed to the twelve farthest nodes from the source [18].

\subsection{Performance Metrics}

The comparison study among SDBP, SFP and FDP is carried out with respect to four performance metrics.

1. Dissemination time: the required time in order that all nodes in the network receive the packet.

2. Packet delivery ratio: the number of nodes that have successfully received the packet divided by the total number of nodes in the network.

3. Forwarders ratio: the percentage of nodes that participated in the rebroadcast operation of the packet. 
4. Redundant packets ratio: the percentage of duplicated packets received with respect to the total number of packets.

5. Number of dropped packets: number of packets discarded at the physical layer by virtue of the following motives [21]:

- Transmission Busy (TXB): this scenario occurs when a node in a transmission state receives another packet. Because a node can not send and receive at the same time, Therefore the packet which was supposed to be received will be dropped.

- Reception Busy (RXB): in this case, a node receives a second packet while it is occupied by the reception of another one. This situation which will cause a collision.

- Searching valid preamble (SXB): in this situation, the node which is in IDLE state drops the packet because it is searching for a valid preamble.

- Receiving a frame preamble (PXB): as the previous case, the node is in IDLE state but it can not receive correctly the current packet because it is busy by receiving a valid preamble of another one.

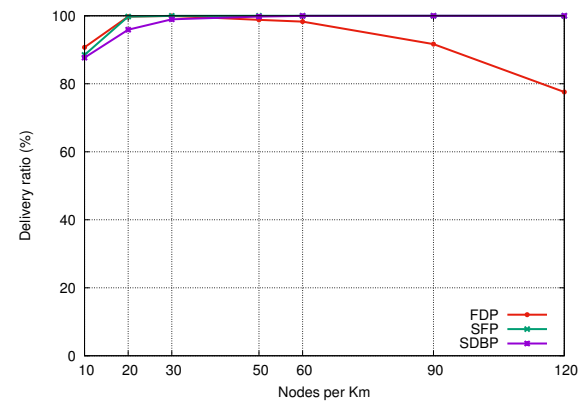

Fig. 4. Packet delivery ratio versus vehicle's density

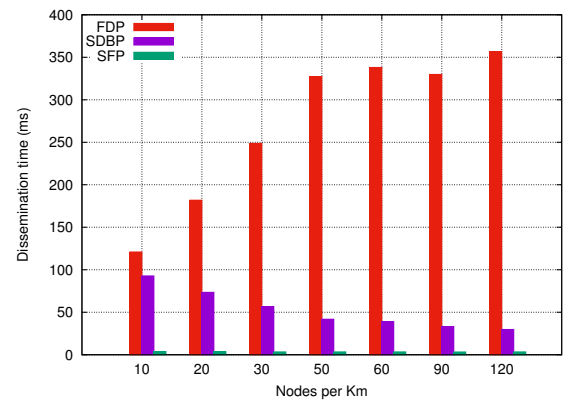

Fig. 5. Dissemination time versus vehicle's density

\subsection{Simulation Analysis}

Figure 4 shows the packet delivery ratio against the vehicle's density. Obtained results indicate that in a low density network the three protocols give roughly the same delivery ratio with a moderate rise of FDP and SFP. When the network starts to be crowded, the ratio reception of FDP falls, because of the loss of packet by the selected farthest nodes. However, SDBP retains a high reachability as SFP whether is a low-density or high-density network.

In terms of dissemination time, figure 5 shows that SDBP outperforms plenty the FDP protocol. As we can see, FDP's dissemination time increases proportionally with vehicle's density owning to the long waiting time of nodes nearby 


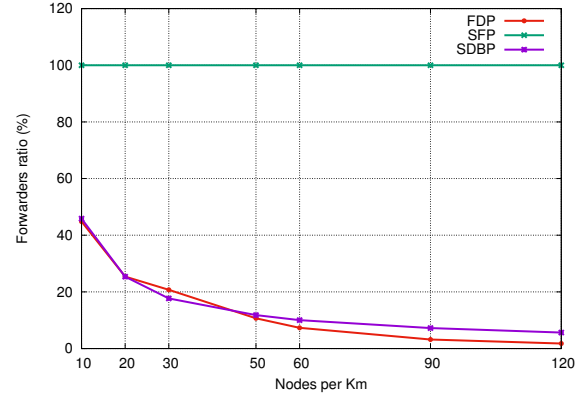

Fig. 6. Forwarders ratio versus vehicle's density

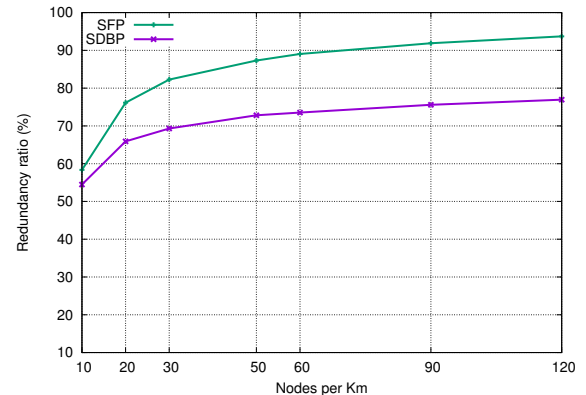

Fig. 7. Redundancy ratio versus vehicle's density

to the source. Unlike the SDBP where the dissemination time decreases proportionately with vehicle's density, because nodes tune their waiting time regularly if no reception is recorded, which allow to the nodes with a short distance to the source to rebroadcast quickly. As expected the SFP presents the smallest values since all nodes rebroadcast the received packets immediately without any delay.

Figure 6 compares the performance of the three protocols in terms of forwarders ratio. Clearly, SFP represents the highest rate (100\%), seeing that all receiver nodes are effective forwarders. Both protocols SDBP and FDP exhibits approximately the same rating from 10 to 50 vehicles $/ \mathrm{km}$. After that, we observe that SDBP slightly overshoots the FDP because SDBP performs better that FDP with regard to the reception ratio.

For a fair comparison in terms of redundancy ratio, we have taken into account the protocols that present a complete reception in the network. As we have shown in Figure 4, when the network become dense, SFP and SDBP always give a delivery ratio of $100 \%$ while FDP's reception rate falls down. Figure 7 shows that SDBP leads to a few number of redundant packets compared to SFP. It should be noted that a certain degree of redundancy must be maintained to ensure a good reception rate.

Figures 8, 9 and 10 show the number of dropped packets for SFP, SDBP and FDP owning to TXB, RXB, SXB and PXB causes. As is foreseen, SFP is ranked first in the packet loss and this for the four reasons. Although FDP gives better results in terms of the number of dropped packets compared with SDBP and $\mathrm{SFP}$, it has the lowest delivery ratio and the highest dissemination time which are key parameters of messages in safety applications.

\section{Conclusion}

This paper introduced a novel efficient broadcasting protocol called SDBP, which allows delivering emergency messages in vehicular safety applications. The purpose is to achieve a high performance in terms of reachability and dissemination time in both sparse and dense network. For that, a hybrid technique which blends 


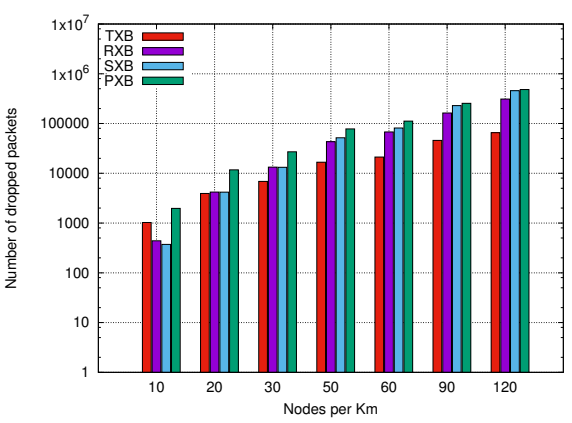

Fig. 8. Number of dropped packets of SFP Fig. 9. Number of dropped packets of versus vehicle's density

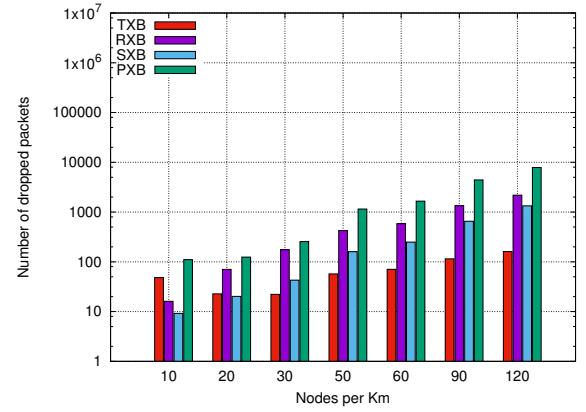

SDBP versus vehicle's density

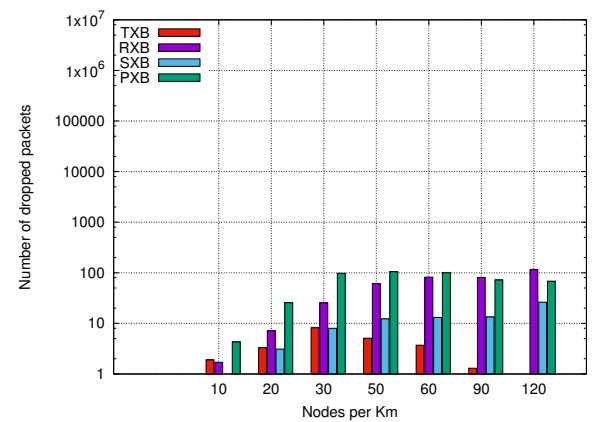

Fig. 10. Number of dropped packets of FDP versus vehicle's density

segment and delay dissemination methods is used. Simulations results show that SDBP outperforms two well-known protocols SFP and FDP. SDBP's good performances are mainly due to some mechanisms like the density-based segmentation and the dynamic adjustment of the waiting time. These mechanisms allow, respectively, a wide coverage of the network and a small dissemination time. The current version of SDBP uses a distance-based to compute the waiting time. In future work, it may be interesting to look at the benefit of adapting the waiting time in accordance with the network density and consider a real road topology in the evaluation process.

\section{References}

1. R. Naja et al., Wireless vehicular networks for car collision avoidance. Springer, 2013, vol. 2013.

2. M. Javadi, S. Habib, and M. Hannan, "Survey on inter-vehicle communication applications: Current trends and challenges," Information Technology Journal, vol. 12 , no. 2, p. 243, 2013.

3. Y.-C. Tseng, S.-Y. Ni, Y.-S. Chen, and J.-P. Sheu, "The broadcast storm problem in a mobile ad hoc network," Wireless networks, vol. 8, no. 2-3, pp. 153-167, 2002. 
4. O. K. Tonguz, N. Wisitpongphan, and F. Bai, "Dv-cast: A distributed vehicular broadcast protocol for vehicular ad hoc networks," IEEE Wireless Communications, vol. 17, no. 2, pp. 47-57, 2010.

5. W. Peng and X.-C. Lu, "On the reduction of broadcast redundancy in mobile ad hoc networks," in Proceedings of the 1st ACM international symposium on Mobile ad hoc networking \& computing. IEEE Press, 2000, pp. 129-130.

6. F. J. Ros, P. M. Ruiz, and I. Stojmenovic, "Reliable and efficient broadcasting in vehicular ad hoc networks," in Vehicular Technology Conference, 2009. VTC Spring 2009. IEEE 69th. IEEE, 2009, pp. 1-5.

7. K. N. Nakorn and K. Rojviboonchai, "Poca: Position-aware reliable broadcasting in vehicular ad-hoc networks," in Proceedings of 2010 Second Asia-Pacific Conference on Information Processing (APCIP 2010), 2010.

8. N. Wisitpongphan, O. K. Tonguz, J. S. Parikh, P. Mudalige, F. Bai, V. Sadekar et al., "Broadcast storm mitigation techniques in vehicular ad hoc networks," IEEE Wireless Commun., vol. 14, no. 6, pp. 84-94, 2007.

9. A. Wegener, H. Hellbruck, S. Fischer, C. Schmidt, and S. Fekete, "Autocast: An adaptive data dissemination protocol for traffic information systems," in Vehicular Technology Conference, 2007. VTC-2007 Fall. 2007 IEEE 66th. IEEE, 2007, pp. 1947-1951.

10. Y. Mylonas, M. Lestas, A. Pitsillides, P. Ioannou, and V. Papadopoulou, "Speed adaptive probabilistic flooding for vehicular ad hoc networks," IEEE Transactions on Vehicular Technology, vol. 64, no. 5, pp. 1973-1990, 2015.

11. I. Achour, T. Bejaoui, A. Busson, and S. Tabbane, "Sead: A simple and efficient adaptive data dissemination protocol in vehicular ad-hoc networks," Wireless Networks, vol. 22, no. 5, pp. 1673-1683, 2016.

12. S. Lima, D. Larces, C. Júnior, and J. Larces, "E-probt: a new approach to mitigate the broadcast storm problem in vanets," in Proceedings of the 31st Annual ACM Symposium on Applied Computing. ACM, 2016, pp. 709-715.

13. G. Korkmaz, E. Ekici, F. Özgüner, and Ü. Özgüner, "Urban multi-hop broadcast protocol for inter-vehicle communication systems," in Proceedings of the 1st ACM international workshop on Vehicular ad hoc networks. ACM, 2004, pp. 76-85.

14. E. Fasolo, R. Furiato, and A. Zanella, "Smart broadcast algorithm for intervehicular communication," in Proceedings of, 2005.

15. J. Sahoo, E. H. K. Wu, P. K. Sahu, and M. Gerla, "Bpab: Binary partition assisted emergency broadcast protocol for vehicular ad hoc networks," in Computer Communications and Networks, 2009. ICCCN 2009. Proceedings of 18th Internatonal Conference on. IEEE, 2009, pp. 1-6.

16. W. B. Jaballah, M. Conti, M. Mosbah, and C. E. Palazzi, "Fast and secure multihop broadcast solutions for intervehicular communication," IEEE Transactions on Intelligent Transportation Systems, vol. 15, no. 1, pp. 433-450, 2014.

17. C. E. Palazzi, M. Roccetti, and S. Ferretti, "An intervehicular communication architecture for safety and entertainment," IEEE Transactions on Intelligent Transportation Systems, vol. 11, no. 1, pp. 90-99, 2010.

18. O. Rehman, M. Ould-Khaoua, and H. Bourdoucen, "An adaptive relay nodes selection scheme for multi-hop broadcast in vanets," Computer Communications, vol. 87, pp. 76-90, 2016.

19. N. Simulator, "ns-2," http://www.isi.edu/nsnam/ns/.

20. SUMO, https://sourceforge.net/projects/sumo/, (2017).

21. A. Hassan, M. H. Ahmed, M. Rahman et al., "Ieee 802.11 p performance evaluation in a city environment," 2011. 